\title{
Shape memory polymer nanofibers and their composites: electrospinning, structure, performance, and applications
}

\author{
Fenghua Zhang ${ }^{1}$, Zhichun Zhang ${ }^{1}$, Tianyang Zhou' ${ }^{1}$, Yanju Liu' ${ }^{2}$ and Jinsong Leng ${ }^{1 *}$ \\ ${ }^{1}$ Centre for Composite Materials and Structures, Harbin Institute of Technology, Harbin, China, ${ }^{2}$ Department of Astronautical \\ Science and Mechanics, Harbin Institute of Technology, Harbin, China
}

OPEN ACCESS

Edited by:

Ilkwon Oh,

Korea Advanced Institute of Science and Technology,

South Korea

Reviewed by:

Naba Kumar Dutta,

Indian Institute of Technology, India Hyacinthe Randriamahazaka,

Université Paris Diderot, France

*Correspondence: Jinsong Leng,

Centre for Composite Materials and Structures, Harbin Institute of Technology (HIT), No. 2 YiKuang Street, PO Box 3011 , Harbin 150080, China lengjs@hit.edu.cn

Specialty section: This article was submitted to Smart

Materials, a section of the journal Frontiers in Materials

Received: 10 April 2015 Accepted: 07 September 2015 Published: 26 October 2015

Citation:

Zhang F, Zhang Z, Zhou T, Liu Y and Leng J (2015) Shape memory polymer nanofibers and their composites: electrospinning, structure, performance, and applications.

Front. Mater. 2:62.

doi: 10.3389/fmats.2015.00062
Shape memory polymers (SMPs) have been defined as a kind of smart materials under great investigation from academic research to industry applications. Research on SMPs and their composites now incorporates a growing focus on nanofibers which offers new structures in microscopic level and the potential of enhanced performance of SMPs. This paper presents a comprehensive review of the development of shape memory polymer nanofibers and their composites, including the introduction of electrospinning technology, the morphology and structures of nanofibers (non-woven fibers, oriented fibers, core/shell fibers, and functional particles added in the fibers), shape memory performance (thermal and mechanical properties, stimulus responsive behavior, multiple and two-way shape changing performance), as well as their potential applications in the fields of biomedical and tissue engineering.

Keywords: shape memory polymers, nanofibers, electrospinning, structure, applications

\section{Introduction}

Since the discovery of shape memory polymers (SMPs) in 1980s, they have the capability to change sizes, shapes, stiffness, or strain when triggered by an external stimulus (heat, electric and magnetic field, water, or light) (Huang et al., 2005; Lendlein et al., 2005; Lan et al., 2009; Liu et al., 2009; Leng et al., 2011; Kumar et al., 2012). SMPs and their composites have captured great attention due to their excellent features, such as multi-shape memory effect, low cost, easy processing, and large deformation (Liu et al., 2007; Xie, 2010; Leng et al., 2011). These unique characteristics guarantee a broad range of applications (Behl and Lendlein, 2007; Liu et al., 2007; Leng et al., 2009, 2011), including actuating, sensing, remote control, smart textiles, robotics and biomedical tissue, as well as aerospace, and so on (Gall et al., 2002; Lendlein and Langer, 2002; Nagahama et al., 2009; Hu et al., 2012; Liu et al., 2014). However, the simple structure limits the development of shape changing materials and practical application. The various fabrication processes result in a diversity of structures of smart polymers. From a structural view point, SMPs can be classified as shape memory blocks, shape memory foams, shape memory fibers, and shape memory films (Leng et al., 2011; Hu et al., 2012). Academic and industrial research on shape memory nanofibers reap huge fruits in the field of fundamental and applied science. Electrospinning method has enjoyed great attention since the 1990s, which is extensively applied to produce nanofibers and their composites. A number of multiple functional materials have been electrospun into nanofibers used in different application fields. SMPs in the form of fibers are generating great 
interest in structural and functional applications because they have well-developed surface area, porous structure, and are easy adsorption, filtration, and penetration, as well as deformation (Cha et al., 2005; Ji et al., 2006; Kotek, 2008; Zhuo et al., 2008a; Chung et al., 2011).

Based on the above advantages, SMPs and their composites with fibrous structure have been increasingly investigated. In recent years, some researches on shape memory nanofibers fabricated by electrospinning have been reported (Meng et al., 2007; Zhuo et al., 2008b; Zhang et al., 2013a, 2014a). Cha et al. synthesized shape memory polyurethane (SMPU) block copolymers that were electrospun into nanofibers at 2005 (Cha et al., 2005). $\mathrm{Hu}$ et al. of the Hong Kong Polytechnic University fabricated the smart polyurethane fibers with shape memory effect (Ji et al., 2006; Zhuo et al., 2008a). Then, the same group further demonstrated that the non-woven SMPU nanofibers (the diameter in a range of $50-700 \mathrm{~nm}$ ) can be triggered by heat and the shape recovery was 98\% after several cycles (Zhuo et al., 2011a). Afterwards, Jing-Nan et al. found that, compared to the bulk SMPU film, SMPU microfibers showed a much quicker shape recovery process when heated in a water bath. The shape recovered from 10 to $90 \%$, the microfiber film only took one-fourth of the time of that needed for the bulk film (Zhang et al., 2011). More recent studies by Lendlein et al. verified that microscaled non-woven fabrics prepared by electrospinning from a chloroform solution of a degradable multiblock copolymer (PDLCL) consisting of crystallizable poly(x-pentadecalactone) hard segments (PPDL) and poly(e-caprolactone) switching segments (PCL) exhibited excellent shape memory performance, with potential application in biomedicine (Matsumoto et al., 2012). An electrospun SMP film with a stable micro/nano-fibrous structure was stable and reversible for at least three cycles of shape memory tests (Chen et al., 2012). In addition, some novel shape memory fibrous composites are being fabricated by adding some functional fillers or synthesized by some special groups can realize the reversible shape changing, two-way and multiple shape memory effect, multiple stimulus actuation, etc. (Gong et al., 2012; Zhang et al., 2013b). Nanotechnology enables SMPs to be utilized in a slice of particular application fields, such as biomedical devices, textiles, nanosensors, and nanoactuators. Most widely recognized potential of SMPs is for artificial blood vessels, drug release, scaffolds, and cell growth template (Mather and Luo, 2011; Mather et al., 2014). New advances in shape memory polymer nanofibers (SMPNs) exhibit a trend of broader applications of small size and flexible materials.

Shape memory fibers in micro- or nano-size are smart, soft, portable, and wearable, promoting the academic and industrial research. This review is mainly seeking to present and discuss the current systematic and comprehensive advancement of electrospun shape memory nanofibers, exhibit ongoing application studies, and provide future perspective. To stimulate further research on smart polymer nanofiber, the content of this work covers the fabrication methods-electrospinning, characterizations, morphologies and structures of electrospun nanofibers, dual- and multi-shape memory effect, thermal-mechanical shape memory cycle, reversible and multifunctional properties, as well as potential applications.

\section{Electrospinning Technology}

Nanofibers are a kind of one-dimensional material, due to these special features mentioned above, and have attracted great attention. Current fiber formation processes include melt spinning, dry spinning, wet spinning, gel spinning, emulsion spinning, shear spinning, and electrospinning. Electrospinning technology, with several unique advantages compared with other methods, has been recognized as the most high-efficiency way to electrospin polymers into the continuous and direct nanofibers (Huang et al., 2003). At present, more than 100 polymers have been successfully fabricated into fibers. The advantages of electrospinning, including low cost, easy processing, and the desirable features for resulting nanofibers such as small diameter, various structures, as well as multifunction usages, mean that electrospun nanofibers have potential applications in biomedical engineering (drug release, hemostatic gauze, etc.), tissue engineering (such as artificial vessels, scaffolds, etc.), textiles, nanosensors, filters (Andreas and Joachim, 2007).

The improvement of electrospinning equipment has led to the nanofibers with desirable structures and properties. The traditional needle electrospinning equipment that contains three parts, including a syringe needle, high voltage power supply, as well as a collector, has been used to prepare nanofibers quite extensively ( $\mathrm{Li}$ and Xia, 2004). According to the experimental results, some theories and models have been developed to investigate the electrospinning process. The quintessential illustration of the electrospinning setup is shown in Figure 2 in Li and Xia, 2004. During the electrospinning process, the polymer solution drop overcomes the surface tension to form the jet when the electrical field is strong enough. Under the electrostatic interaction, the droplet is distorted into a conical shape named the Taylor cone. The jet flow is drawn into a straight line, and then curves to the spiral (Reneker and Chun, 1996). The electrostatic force induces the jet flow to be stretched and divided into ultrathin fibers. At last, the nanofibers are obtained on the collector after the solvent evaporating. On macro-scale, electrospinning, eventually, generates a fibrous network membrane. The applied voltage, distance between tip and collector, solution concentration, and flow rate, as well as viscosity and conductivity of polymers, have a critical influences on electrospinning results (Shin et al., 2001). The various structures and diameters of fibers can be designed by changing the electrospinning conditions. The electrospinning process is usually elaborately controlled and modified to meet the practical requirements.

\section{Morphology and Structure of SMP Nanofibers}

It is worth pointing out that shape memory fibers based on SMPs and their composites are fabricated by electrospinning technology, which exhibit different structures and morphologies based on the processing parameters. The affect factors include the changes in the solution concentration, applied electric field, spinneret structure, the distance between spinneret and collector, as well as the collectors (Deitzel et al., 2001; Li and Xia, 2004). In addition to the above mentioned factors, temperature and humidity are also 
important factors in determining the morphology and diameters of electrospun fibers. Research papers and patents demonstrate that nanofibers obtained through electrospinning with some special morphology and structures can be synthesized if the appropriate electrospinning processing conditions are employed. Hu et al. have discussed the dependence of the morphology of SMPU nanofibers on the applied voltage, solution concentration, and feeding rate. In particular, the solution concentration played an important role in transforming the polymer solution into ultrafine fibers. In general, increased concentration will lead to increased fiber diameters (Meng et al., 2007).

The non-woven fibers are the most simple and traditional structure, the porous morphology can be programmable during the shape changing process (Deitzel et al., 2001; Matsumoto et al., 2012). Figure 2 in Zhuo et al. (2011a) shows the morphology of the typical non-woven nanofibers examined by SEM, the diameters of which vary from 600 to $700 \mathrm{~nm}$. With the use of a similar electrospinning setup, other researchers also get the same structure when the various polymers are electrospun into fibers (Chen et al., 2012; Gong et al., 2012; Zhang et al., 2013b). Nonwoven fibrous structure shows an excellent pore network and can be used as filters or catalysts.

For practical applications, such as photonic or electrical products, as well as fiber reinforced composites, a number of polymers are indispensable. These are then made into the well-oriented and highly ordered fibers (Kameoka et al., 2003). Various approaches are used to fabricate the aligned fibers. As an example, some researchers modified the electrospinning collectors into rotating drum or a pair of split electrodes as collectors for forming the aligned fibers (Andreas and Joachim, 2007). The resulting fibers formed by different collectors show varied degrees of orientation. Some oriented fibers exhibiting excellent shape memory effect have been prepared. Ji et al. collected SMPU fibers with oriented morphology (Figure 1) (Ji et al., 2006). It was found that the smart fibers exhibited excellent recovery for application of mechanical force, to an extreme extent, providing a possibility to generate the actuators with high-performance and high recovery stress. It is necessary to control the morphology and structure that accelerate the development of smart nanofibers. Such architectures will be useful in improving shape recovery and shape fixity behaviors.

In addition, the specific secondary structure, such as core/shell, has been fabricated by designing the spinneret comprising two coaxial capillaries (Sun et al., 2003; Rana et al., 2013). SMPU fibers with core/shell nanostructure prepared by co-electrospinning

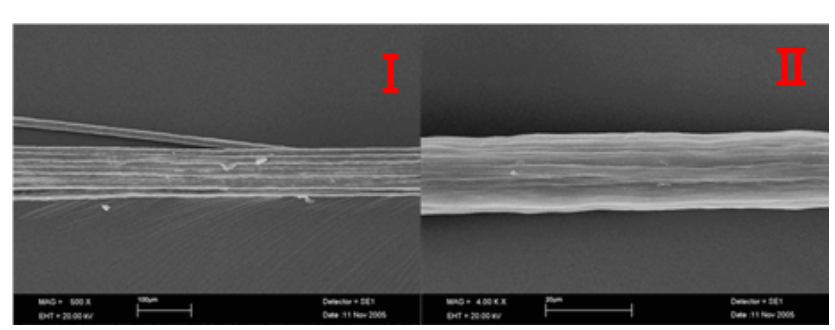

FIGURE 1 | SEM of SMF-3. View of multifilament (500x) (Ji et al., 2006 (C) IOP Publishing. Reproduced with permission. All rights reserved). two different concentration solutions have been demonstrated (Zhuo et al., 2011b). As shown in Figure 3 in Zhuo et al. (2011b) transmission electron microscopy (TEM) images show the beadon-string structure (I) and core-shell structure (II), obtained from the core solution of polycaprolactone-based SMPU and shell solution of pyridine-containing polyurethane. This kind of composite nanofibers with antibacterial activity was proposed to be used as smart materials and antibacterial materials. Historically, electrospinning was limited to the feature of polymer, which means that some kinds of polymers cannot be electrospun into fibers due to their poor spinnability. To this end, our group has recently fabricated the PCL/epoxy composite fibers with core/ shell structure by co-electrospinning two polymers that phase separate (Zhang et al., 2014b, 2015). Co-electrospinning serving as a template provides an easy way to make the polymers without spinnability into fibrous structure, broadening the applications in micro- or nano-scale devices.

Besides the fibers with special structures, some multifunctional fibers filled with functional materials, such as carbon nanotubes, carbon blacks, carbon nanofibers, and magnetic particles $\left(\mathrm{Fe}_{3} \mathrm{O}_{4}\right.$, Ni nanowires, etc.), have been developed (Leng et al., 2007; Sahoo et al., 2010; Zhang et al., 2014c). Introduction of nanoparticles into polymers for electrospinning to generate well-defined properties has been demonstrated. For example, incorporation of $\mathrm{Fe}_{3} \mathrm{O}_{4}$ into polycaprolactone (PCL) nanofibers has been reported (Gong et al., 2012). The results indicated that the composite fibers were able to be triggered by alternating magnetic field, which was an easy method for implementing remote control. Furthermore, the Alamar blue assay suggested that the electrospun composite fibers possessed good biocompatibility and could be applied in biomedical fields.

\section{Shape Memory Performance}

As seen in the increasing number of various research papers and patents, research on shape memory behaviors of SMPNs has captured worldwide attention (Mather and Luo, 2011; Sauter et al., 2012; Mather et al., 2014). Other properties of SMPNs, which also affect the shape memory effect, have been investigated. The thermal, mechanical, and chemical properties of SMPNs are characterized by differential scanning calorimetry (DSC), thermogravimetric analysis (TGA), infrared camera, dynamic mechanical analysis (DMA), mechanical tension test, Fourier transform infrared spectroscopy (FTIR), and so on (Leng et al., 2011). DMA and mechanical testing are also used to measure the shape memory performance of SMPNs. And the scanning electron microscopy (SEM) and TEM were carried out to observe the morphology and structure of SMPNs. Recently, in order to analyze the mechanisms of shape memory effect based on the shape changing of the internal microstructures of electrospun fibers, the microscopic deformation was conducted.

Leng's group has reported the microscopic shape changes. As shown in Figure 2, the microscopic shape memory behavior of electrospun Nafion nanofiber membrane could be programmable (Zhang et al., 2014a). At a microscopic level, the original nonwoven fibrous structure was easily deformed to the temporary shape, and then recovered its original structure when the sample 


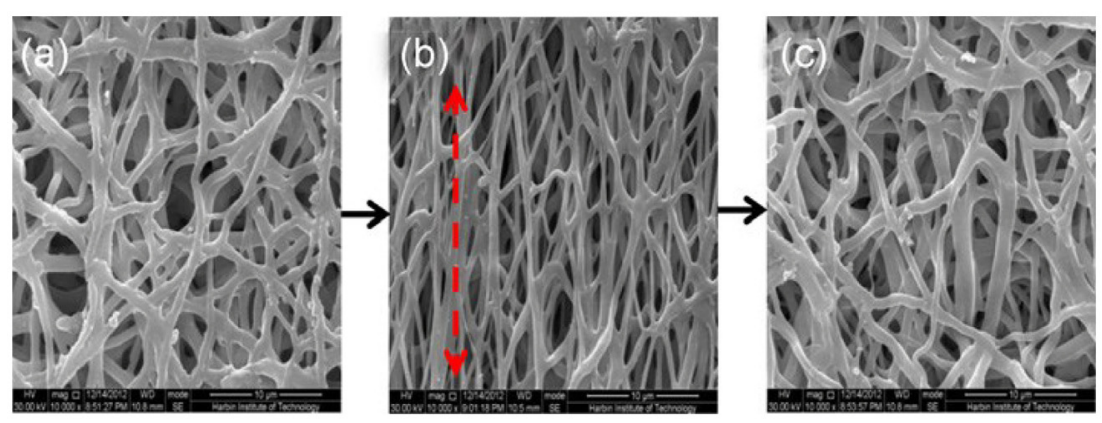

FIGURE 2 | SEM images of Nafion nanofibers, (a) the original fibers, (b) the stretched fibers, and (c) recovered fibers. (With kind permission from Springer Science+Business Media Zhang et al., 2014a).

was reheated. This result implied that the nanofibers' structure was stable after cycles of shape memory test. The same phenomenon was also studied (Zhuo et al., 2011a). Furthermore, Chen et al. have reported a kind of polyurethane nanofibers showing reversible shape memory effect (Zhang et al., 2011). The fibers showed different structures before and after deformation. After the fibrous film was stretched and fixed, the fibers tended to orientate along the strain direction. The orientated fibers were actuated to random form when the macro-film recovered the original shape in each cycle.

Other performances and variations for SMP fibers could be achieved by designing molecular ratios. Bao et al. have demonstrated that they have successfully fabricated a kind of biomedical fibrous scaffolds, made from biodegradable poly(D,L-lactide-cotrimethylene carbonate) fibers with shape memory effect (Bao et al., 2014). The monomer ratio of DLLA: TMC varied from 5:5 to $9: 1$, which resulted in the glass transition temperature $T_{g}$ of the fibrous scaffolds to increase from 19.2 to $44.2^{\circ} \mathrm{C}$. As shown in Figure 3, the shape memory effect of fibrous PLMC scaffolds in the form of spiral and cylindrical bar are successfully proved. The spiral shape was able to recover to the strip shape when dipped in the water bath at $39^{\circ} \mathrm{C}$ (Figure 3A). The macroscopic deformation in the 3-D shapes of "S," "M," and "P" completely recovered quickly to their original shapes with a speed of $12 \mathrm{~s}$ at $39^{\circ} \mathrm{C}$ (Figure $3 \mathrm{~B}$ ). The densely packed and partially fused fibrous structure influenced the fibers in contact with the hot surrounding because the response rate is dependent on the heat transfer rate. The rapid shape recovery process of these electrospun fibers were attributed to their high specific surface area. Therefore, SMPNs with fibrous structure were more suitable to be used as the immediate activation or constructing devices due to a long activation time. The PLMC fibers exhibited outstanding shape fixity and shape recovery properties with both shape recovery and shape fixity ratios above $90 \%$.

The typical programing of a thermomechanical cycle test for bulk SMPs can be carried out to investigate the shape memory properties of SMPNs. The dual-shape memory behavior of thermo-induced SMPNs is presented in Figure 4, consisting of two steps (shape fixity and shape recovery) and showing the effect of temperature on the strain and the stress (Zhang et al., 2014a). The electrospun nanofiber membrane was heated at $130^{\circ} \mathrm{C}$ for $10 \mathrm{~min}$, then cooled down to $40^{\circ} \mathrm{C}$. During this process, an external force was applied to the sample inducing the change in strain from 0 to $56 \%$. This was the shape fixing step. When the membrane was exposed at $40^{\circ} \mathrm{C}$ for a certain time, it could recover its original shape after reheating above the transition temperature $\left(130^{\circ} \mathrm{C}\right)$, which was defined as shape recovery process. The electrospun Nafion nanofibers showed perfect dual-shape memory property when deformed and recovered at $130^{\circ} \mathrm{C}$. Equations 12 are used to calculate the shape fixity ratio $\left(R_{f}\right)$ and shape recovery ratio $\left(R_{r}\right)$ of SMPNs (Xie, 2010). The $R_{f}$ and $R_{\mathrm{r}}$ were all above $90 \%$ when triggered by heat.

$$
\begin{aligned}
& R_{\mathrm{f}}(X \rightarrow Y)=\frac{\varepsilon_{\mathrm{y}}-\varepsilon_{\mathrm{x}}}{\varepsilon_{\mathrm{y}, \text { load }}-\varepsilon_{\mathrm{x}}} \times 100 \% \\
& R_{\mathrm{r}}(Y \rightarrow X)=\frac{\varepsilon_{\mathrm{y}}-\varepsilon_{\mathrm{x}, \text { rec }}}{\varepsilon_{\mathrm{y}}-\varepsilon_{\mathrm{x}}} \times 100 \%
\end{aligned}
$$

where $X$ and $Y$ denoted different shapes, $\epsilon_{y}$ load was the maximum strain under load, $\epsilon_{y}$ and $\epsilon_{x}$ were the fixed strains after cooling and load removal, and $\epsilon x$,rec was the strain after recovery.

In order to discuss the repeatability and durability, the shape memory cycles of SMPNs should be tested more than once. For instance, the electrospun non-woven fibers were fabricated from degradable polyesterurethanes named PDLCL containing poly(x-pentadecalactone) hard segments (PPDL) and poly(ecaprolactone) switching segments (PCL), demonstrating excellent shape memory properties. The strain-stress curves of the thermomechanical cycles are shown in Figure 4 in Matsumoto et al. (2012), which describes the shape memory cycles over three times. As it can be seen from this figure, compared to the first cycle, the shape recovery ratio of the second cycle deduces to $89 \%$. However, the shape fixity ratios in all cycles stay constant at $83 \%$. These results suggested that the electrospun PDLCL fibers enjoyed favorable reproducible shape-memory properties. Matsumoto et al., 2012 also describes the four subsequent cycles of shape memory test, the $\epsilon_{\mathrm{m}}$ increases from 25 to $50 \%$. The shape recovery ratio was lower than the aforementioned experiments when $\epsilon_{\mathrm{m}}$ was $25 \%$ after the second cycle. The stress showed linear increase until the strain reached the last cycle value, and then the stress relaxation was generated, which gave rise to the recovery rate decrease. 
A
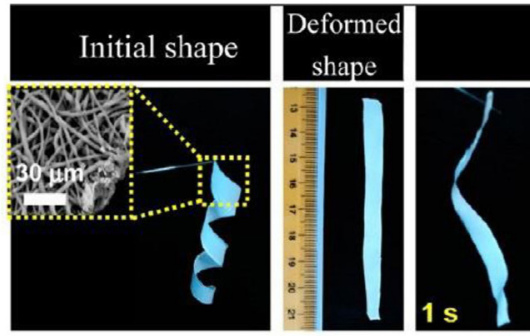

Shape recovery process

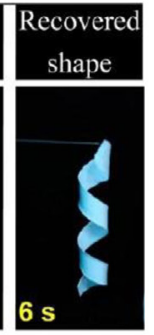

B
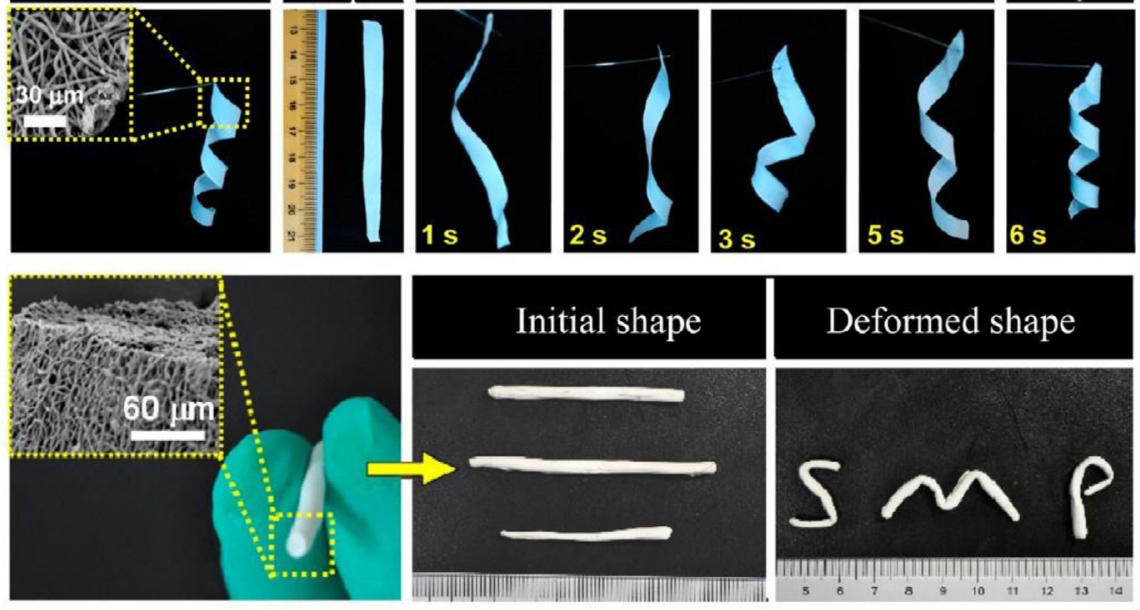

Shape recovery process

\section{Recovered shape}
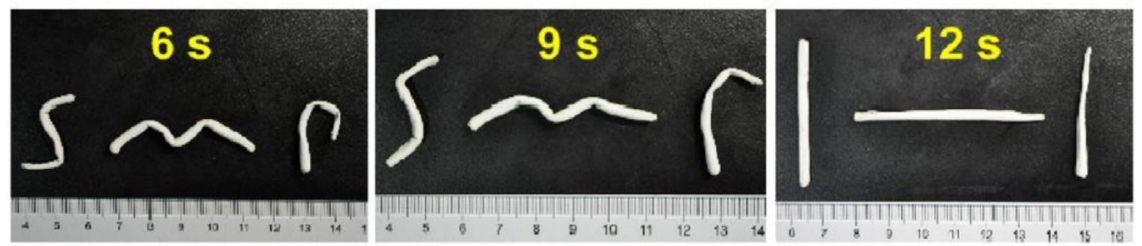

FIGURE 3 | Macroscopic demonstration of the shape memory effect for electrospun fibrous PLMC. (A) Shape recovery process of a spiral in membranous form (2-D); (B) shape recovery process of the cylindrical bars made of electrospun fibers (inset image), in transition from the temporary shape ("SMP") to the permanent straight bars. Reprinted with permission from Bao et al. (2014). Copyright (2014) American Chemical Society.

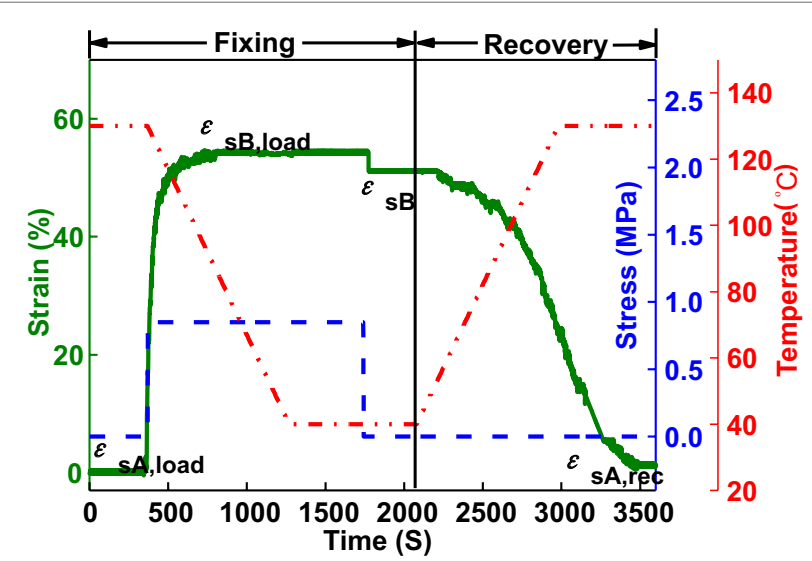

FIGURE 4 | Shape memory cycle test. (With kind permission from Springer Science+Business Media: Zhang et al., 2014a)

Expanding the multifunction of SMPNs, researchers design the special methods to stimulate the shape recovery processes. Zhou et al. found that the electrospun spider silk protein had a crystallization memory property when it was stimulated by a high pressure (Peng et al., 2009). Furthermore, the moisture-responsive Kraft lignin-based materials prepared by electrospinning blends of Kraft lignin fractions with different physical properties are reported, as shown in Figure 5. Moreover, the different material morphologies, including submicrometer fibers, bonded non-wovens, porous films, and smooth films were activated by the differences in thermal mobility between lignin fractions influencing the degree of inter fiber fusion occurring during oxidative thermostabilization of electrospun non-woven fabrics (Dallmeyer et al., 2013). The size of nanopores/micropores in SMPU membranes prepared by electrospinning with two-way shape memory effect was investigated successfully. According to the temperature change, the pore size can be changed from 150 to $440 \mathrm{~nm}$, demonstrating that the SMPN membrane can serve as a smart membrane to selectively separate substances by controlling temperature only (Ahn et al., 2011).

We note that simple shape memory behaviors are limited to the practical applications. It has been reported that the electrospun Nafion fibrous membranes can memorize more than two shapes, which is named multiple shape memory effect (Zhang et al., 2013b). Nafion, consisting of polytetrafluorethylene backbone and perfluoroether sulfonic acid side chains, possesses promising properties that give rise to numerous applications of the materials in a variety of fields. Nafion solution with concentration 5\% can be electrospun into nanofibers by addition of a very small amount of poly(ethyleneoxide) (PEO). The resulting fibers, with 


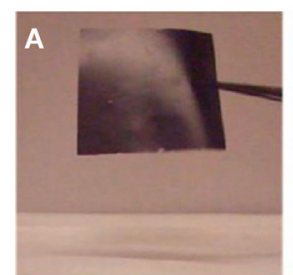

Wet surface

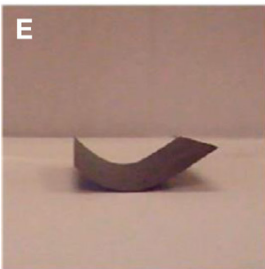

Dry surface, $\mathrm{t}=0$

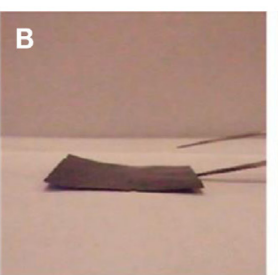

$\mathrm{t}=0$

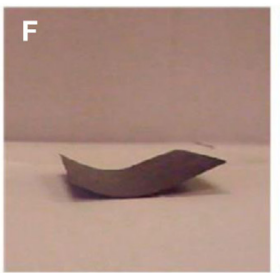

$\mathrm{t}=10 \mathrm{~s}$

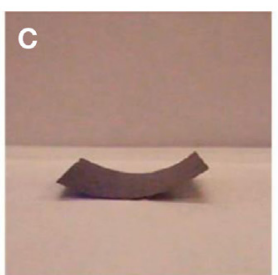

$\mathrm{t}=2 \mathrm{~s}$

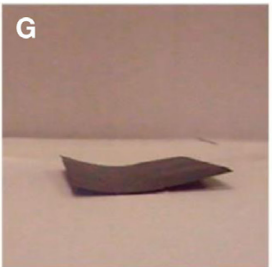

$\mathrm{t}=30 \mathrm{~s}$

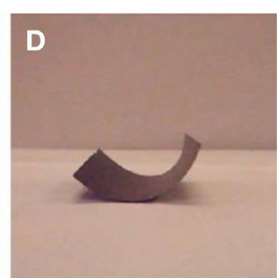

$\mathrm{t}=10 \mathrm{~s}$

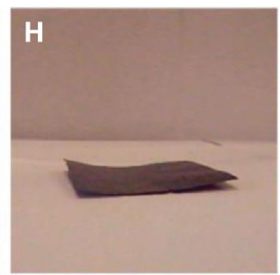

$t=45 s$

FIGURE 5 | Thermostabilized ( $\left.5^{\circ} \mathrm{C} / \mathrm{min}\right) \mathbf{5 0 / 5 0}$ F4/F1-3 film placed on moist paper (A-D), then moved to dry paper (E-H). Reprinted with permission from Dallmeyer et al. (2013). Copyright (2013) American Chemical Society.
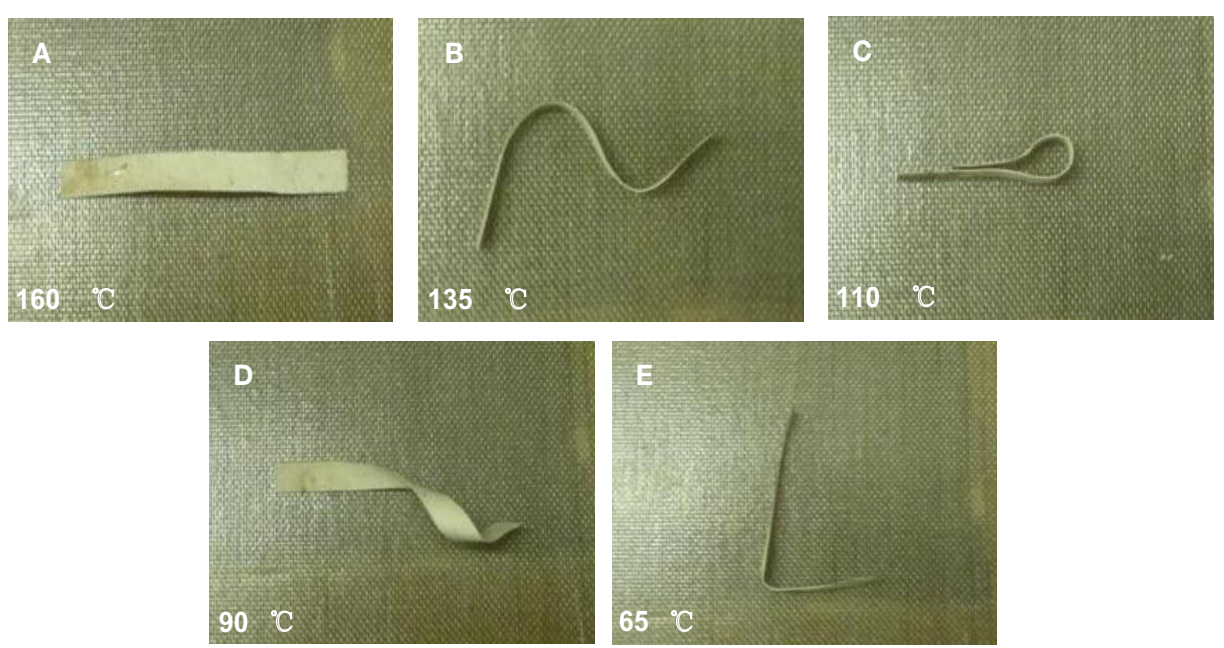

FIGURE 6 | Photos of electrospun Nafion nanofiber membranes with quintuple-shape memory effect. (Zhang et al., 2013b, () IOP Publishing. Reproduced with permission. All rights reserved).

a broad transition temperature range from 60 to $170^{\circ} \mathrm{C}$, show uniform and continuous non-woven structure, which is designed to remember five shapes at different transition temperatures, as shown in Figure 6. The multiple temporary shapes of electrospun Nafion fibrous membranes can be induced by heat in a single triple-, quadruple-, quintuple-shape memory cycle with a high speed, respectively. After the quintuple-shape memory cycle, the samples still keep the stable nanofiber morphology, providing potential applications in smart textiles, sensors, and actuators, as well as artificial skins.

Further efforts to integrate novel functions of SMPNs into applications will likely prove advantages and an important step forward advantage. For instance, multi-stimulus responsive shape memory fibers may remotely control the cell grow in different shape changing template surfaces. Two-way shape memory effect enables fibrous membranes or textiles to change porous structure under tunable conditions, for example, in intelligent clothes. Moreover, improvement to the eletrospinning processing will permit practical applications of nanofibers in the future.

\section{Applications}

SMPs can be fixed into pre-designed shapes and recover their permanent shapes, which can be triggered by heat, moisture, solutions, light, electricity, magnetism, or multi-stimulus. The unique feature provides SMPs' unlimited potential uses. In recent years, SMPNs as a novel kind of smart fibrous materials also have wide ranging applications (Fu et al., 2009; Ko et al., 2013; Chung 
et al., 2014), such as smart fabrics and biomedical materials (e.g., biomimetic scaffolds, cell growth template, self-healing application, medical devices, antibacterial nanomaterials) (Zhuo et al., 2011b; Schneider et al., 2012; Tseng et al., 2012; Zhang and Li, 2013). In particular, shape memory scaffolds with high porosity and specific surface area that have also found potential for use in tissue engineering in the future. The traditionally used structures are static physical structures and poorly suited to mimicking the complex dynamic behavior of in vivo microenvironments. Shape memory nanofibers provide an attractive class of supports for biotechnology and tissue engineering thanks to their large surface areas and small diameters.

An interest in biodegradable and biocompatible SMPs and progress in biotechnology will result in the commercial production of biomedical tissues. For example, the shape memory poly(D,L-lactide-co-trimethylene carbonate) fibers fabricated by electrospinning have potential application as bone scaffolds (Bao et al., 2014). Authors analyzed the proliferation and morphology of osteoblasts cultured onto the electrospun nanofibrous scaffolds as revealed in Figure 7. Nanofibrous scaffolds of PLMC with ratio $(8: 2)\left(\mathrm{Tg}=36.7^{\circ} \mathrm{C}\right)$ and PLMC with ratio $(9: 1)\left(\mathrm{Tg}=44.2^{\circ} \mathrm{C}\right)$ were used to investigate their properties. The cytocompatibility was calculated by MTT assay, the results of which are presented in Figure 7A. The cellular spreading, adhesion, and growth abilities showed similar tendency (Figure 7B). Figure 7C exhibits the SEM images of morphology of osteoblasts attached and proliferated on the nanofibrous PLMC scaffolds. It can be seen from Figure 7D, the cells were stained after 4 and 7 days of culture, in order to monitor the cell morphology of proliferating osteoblasts on the nanofibers. All these results indicated that the electrospun fibrous scaffolds with shape memory effect could be applied in repairing various bone defects, including healing bone crew holes and guided bone regeneration.

Tseng et al. have demonstrated that the electrospun SMPU fiber scaffold is capable of being programed to change macroscopic shape and microscopic architecture during cell culture. This smart fibrous scaffold was adopted to test the hypothesis that a shape-memory-actuated change in scaffold fiber alignment can be used to control the behavior of attached and viable cells, as shown by Tseng et al. (2013). The shape memory triggered changes in scaffold fiber alignment were demonstrated by the attached and viable cells, controlling the cell morphology. Scaffolds, with shape memory effect, that play an important role in the biological fields, are potential to provide in vitro platforms for the investigation of cells and tissues.

Furthermore, some improved and useful SMPs and SMPNs have also been developed. Dye-containing polymers that have potential applications in medical fields, such as medical devices, or drug delivery have attracted a host of researchers. To this end, Torbati et al. have systematically investigated a light-emitting shape memory poly(vinyl acetate) (PVAc) web fabricated by electrospining with indocyanine green (Torbati et al., 2014). As shown in Figure 6 in Torbati et al. (2014) the electrospun webs are cut into different shapes, which are able to shrink when immersed in $25^{\circ} \mathrm{C}$ water or heated at $50^{\circ} \mathrm{C}$, respectively. The feature of water-induced shape changes provides the possible applications in medical devices, including feeding tubes and catheters.

As described in previous sections, SMPNs are remarkably useful and flexible materials for a number of applications. In addition to the fiber reinforced composites, biomedical usages, smart clothes, and smart sensors, SMPNs will expand the usages of smart catalysts, sacrificial templates, and smart electronic devices, as well as smart optical devices in the future. It is expected that research on SMPNs will be deepen and broaden to include interdisciplinary research and technological convergence, generating more smart products to promote the development in smart materials and structures.

\section{Conclusion and Perspective}

In this paper, the various dimensions of SMPNs have been systematically discussed, including the fabrication methods, characterization, structures, properties, and potential applications in broad fields in the future. The electrospinning technology as an effective approach to fabricate nanofibers has been used to obtain the different kinds of SMPNs which show excellent thermal and mechanical properties, as well as shape memory effect after several cycles. The diversity of fibers' structures is mainly based on the electrospinning conditions, including needle's structure, collector, applied voltages, and concentration of polymeric solutions. SMPNs are capable of experiencing a large recoverable and revisable deformation upon some certain stimuli, including heat, magnetic field, electricity, moisture, light, etc. SMPNs and their composites are attractive because of their potential applications, especially in biomedical engineering, including drug release, scaffolds, and cell growth templates in the future.

The merits of SMPNs and their composites include fast response, good flexibility, low weight, easy processing, multiple function, and diverse structures. However, compared with the block SMPs, SMPNs show low strength. Moreover, some of SMPs are not able to be electrospun into fibers. Recently, functional particles, such as carbon nanotubes and carbon blacks, $\mathrm{Fe}_{3} \mathrm{O}_{4}$, have been loaded into the fibers to enhance the mechanical and magnetic properties. In addition, co-electrospinning method, a good choice to form the core/shell fibers, makes some polymers without spinnability into fibers. Furthermore, SMPNs are better candidates for biomedical applications. One of the critical changes is to realize the effective actuation. One possible way to solve this problem is to add some magnetic particles in the fibers to achieve the remote control by alternating magnetic field.

Therefore, based on the previous works, the future direction of SMPN will focus on the following research:

(1) New kinds of SMPN. Prospective SMPN may cover twoway SMPN, thermosetting SMPN, and SMPN with diverse structure, etc.

(2) Multiple stimulus of SMPN. At present, most of SMPN can be triggered by electricity, heat, or moisture, and so on. In order to meet the practical applications, remote stimulus is necessary. And some special fields also require the multistimulus which would enable the materials to tune the stimulus under different conditions. 

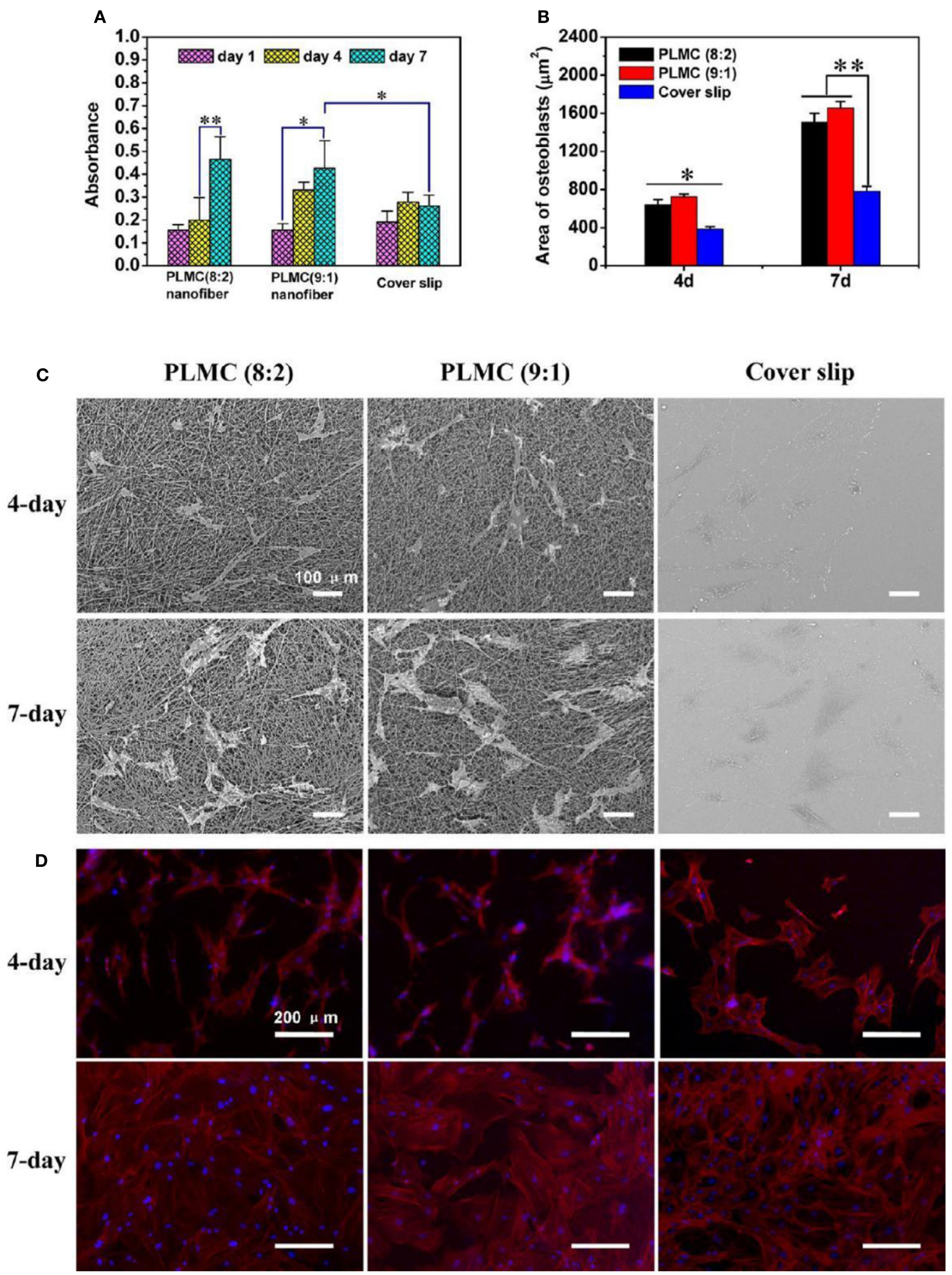

FIGURE 7 | Proliferation and morphology observation of osteoblasts cultured onto the electrospun nanofibrous scaffolds of PLMC (8:2) and PLMC (9:1), and the coverslip control for up to 7 days. (A) Histogram of osteoblast proliferation by MTT assay; (B) histogram of area of osteoblasts; (C) SEM micrographs; (D) fluorescence staining of osteoblasts. F-actin filaments (red) and nucleus (blue) were stained by Phalloidin and DAPI, respectively. Reprinted with permission from Bao et al. (2014). Copyright (2014) American Chemical Society. 
(3) Multifunctional SMPN and broad applications. Except for shape memory effect, some functional materials can be added into the SMPN, which will possibly induce novel functions and applications in the future.

\section{References}

Ahn, J. S., Yu, W. R., Youk, J. H., and Ryu, H. Y. (2011). In situ temperature tunable pores of shape memory polyurethane membranes. Smart Mater. Struct. 20, 105024. doi:10.1088/0964-1726/20/10/105024

Andreas, G., and Joachim, H. W. (2007). Electrospinning: a fascinating method for the preparation of ultrathin fibers. Angew. Chem. Int. Ed. Engl. 46, 5670-5703. doi:10.1002/anie.200604646

Bao, M., Lou, X., Zhou, Q., Dong, W., Yuan, H., and Zhang, Y. (2014). Electrospun biomimetic fibrous scaffold from shape memory polymer of PDLLA-co-TMC for bone tissue engineering. ACS Appl. Mater. Inter. 6, 2611-2621. doi:10.1021/ am405101k

Behl, M., and Lendlein, A. (2007). Shape-memory polymers. Mater. Today 10, 20-28. doi:10.1016/S1369-7021(07)70047-0

Cha, D. I., Kim, H. Y., Lee, K. H., Jung, Y. C., Cho, J. W., and Chun, B. C. (2005). Electrospun nonwovens of shape-memory polyurethane block copolymers. J. Appl. Polym. Sci. 96, 460-465. doi:10.1002/app.21467

Chen, H. L., Cao, X. Y., Zhang, J. N., Zhang, J. J., Ma, Y. M., Shi, G., et al. (2012). Electrospun shape memory film with reversible fibrous structure. J. Mater. Chem. 22, 22387-22391. doi:10.1039/C2JM33970F

Chung, S. E., Park, C. H., Yu, W. R., and Kang, T. J. (2011). Thermoresponsive shape memory characteristics of polyurethane electrospun web. J. Appl. Polym. Sci. 120, 492-500. doi:10.1002/app.33167

Chung, Y. C., Yang, K., Choi, J. W., and Chun, B. C. (2014). Characterization and application of polyurethane copolymers grafted with photoluminescent dyes. Color. Technol. 130, 305-313. doi:10.1007/s12221-014-0008-3

Dallmeyer, I., Chowdhury, S., and Kadla, J. F. (2013). Preparation and characterization of Kraft lignin-based moisture-responsive films with reversible shapechange capability. Biomacromolecules 14, 2354-2363. doi:10.1021/bm400465p

Deitzel, J. M., Kleinmeyer, J., Harris, D., and Beck Tan, N. C. (2001). The effect of processing variables on the morphology of electrospun nanofibers and textiles. Polymer 42, 261-272. doi:10.1016/S0032-3861(00)00250-0

Fu, G. D., Xu, L. Q., Yao, F., Li, G. L., and Kang, E. T. (2009). Smart nanofibers with a photoresponsive surface for controlled release. ACS Appl. Mater. Inter. 1, 2424-2427. doi:10.1021/am900526u

Gall, K., Dunn, M. L., Liu, Y. P., Finch, D., Lake, M., and Munshib, N. A. (2002). Shape memory polymer nanocomposites. Acta Mater. 50, 5115-5126. doi:10.1016/S1359-6454(02)00368-3

Gong, T., Li, W., Chen, H., Wang, L., Shao, S., and Zhou, S. (2012). Remotely actuated shape memory effect of electrospun composite nanofibers. Acta Biomater. 8, 1248-1259. doi:10.1016/j.actbio.2011.12.006

Hu, J. L., Zhu, Y., Huang, H. H., and Lu, J. (2012). Recent advances in shape-memory polymers: structure, mechanism, functionality, modeling and applications. Prog. Polym. Sci. 37, 1720-1763. doi:10.1016/j.progpolymsci.2012.06.001

Huang, W. M., Yang, B., An, L., Li, C., and Chan, Y. S. (2005). Water-driven programmable polyurethane shape memory polymer: demonstration and mechanism. Appl. Phys. Lett. 86, 114105. doi:10.1063/1.1880448

Huang, Z. M., Zhang, Y. Z., Kotaki, M., and Ramakrishna, S. (2003). A review on polymer nanofibers by electrospinning and their applications in nanocomposites. Compos. Sci. Technol. 63, 2223-2253. doi:10.1016/ S0266-3538(03)00178-7

Ji, F. L., Zhu, Y., Hu, J. L., Liu, Y., Yeung, L. Y., and Ye, G. D. (2006). Smart polymer fibers with shape memory effect. Smart Mater. Struct. 15, 1547-1554. doi:10.1088/0964-1726/15/6/006

Kameoka, J., Orth, R., Yang, Y., Czaplewski, D., Mathers, R., Coates, G. W., et al. (2003). A scanning tip electrospinning source for deposition of oriented nanofibres. Nanotechnology 14, 1124-1129. doi:10.1088/0957-4484/14/10/310

Ko, Y. I., Kim, B. S., Bae, J. S., Kim, Y. A., and Kim, I. S. (2013). Silicone-coated elastomeric polylactide nanofiber filaments: mechanical properties and shape memory behavior. RSC Adv. 3, 20091-20098. doi:10.1039/c3ra42361a

\section{Acknowledgments}

This work is supported by the National Natural Science Foundation of China (Grant No. 11225211 and No.11272106).

Kotek, R. (2008). Recent advances in polymer fibers. Polym. Rev. 48, 221-229. doi:10.1080/15583720802020038

Kumar, U. N., Kratz, K., Behl, M., and Lendlein, A. (2012). Shape memory properties of magnetically active triple-shape nanocomposites based on a grafted polymer network with two crystallizable switching segments. Express Polym. Lett. 6, 26-40. doi:10.3144/expresspolymlett.2012.4

Lan, X., Liu, Y. J., Lv, H. B., Wang, X. H., Leng, J. S., and Du, S. (2009). Fiber reinforced shape-memory polymer composite and its application in a deployable hinge. Smart Mater. Struct. 18, 024002. doi:10.1088/0964-1726/18/2/024002

Lendlein, A., Jiang, H., Junger, O., and Langer, R. (2005). Light-induced shape-memory polymers. Nature 434, 879-882. doi:10.1038/nature03496

Lendlein, A., and Langer, R. (2002). Biodegradable, elastic shape-memory polymers for potential biomedical applications. Science 296, 1673-1676. doi:10.1126/ science. 1066102

Leng, J. S., Lan, X., Liu, Y. J., and Du, S. Y. (2011). Shape-memory polymers and their composites: stimulus methods and applications. Prog. Mater. Sci. 56, 1077-1135. doi:10.1016/j.pmatsci.2011.03.001

Leng, J. S., Lv, H. B., Liu, Y. J., and Du, S. Y. (2007). Electroactivate shape-memory polymer filled with nanocarbon particles and short carbon fibers. Appl. Phys. Lett. 91, 144105. doi:10.1063/1.2790497

Leng, J. S., Lv, H. B., Liu, Y. J., Huang, W. M., and Du, S. Y. (2009). Shape-memory polymers - a class of novel smart materials. MRS Bull. 34, 848-855. doi:10.1557/ mrs2009.235

Li, D., and Xia, Y. N. (2004). Electrospinning of nanofibers: reinventing the wheel? Adv. Mater. 16, 1151-1170. doi:10.1002/adma.200400719

Liu, C., Qin, H., and Mather, P. T. (2007). Review of progress in shape-memory polymers. J. Mater. Chem. 17, 1543-1558. doi:10.1039/B615954K

Liu, Y. J., Du, H. Y., Liu, L. W., and Leng, J. S. (2014). Shape memory polymers and their composites in aerospace applications: a review. Smart Mater. Struct. 23, 023001. doi:10.1088/0964-1726/23/2/023001

Liu, Y. J., Lv, H. B., Lan, X., Leng, J. S., and Du, S. Y. (2009). Review of electro-active shape-memory polymer composite. Compos. Sci. Technol. 69, 2064-2068. doi:10.1016/j.compscitech.2008.08.016

Mather, P. T., and Luo, X. F. (2011). Shape Memory Elastomer, Useful e.g. to Form Adaptive Seals Such as Heat-Shrinkable Seals that Prevent Water Leaking and Produce Configurable Surgical Tools, Comprises Non-Woven Mat, and Resin Matrix Infiltrated Throughout the Mat. US2011021097-A1.

Mather, P. T., Torbati, A., and Mather, R. (2014). Near Infrared Fluorescent Marker for Use in e.g. Medical Device During Surgical Procedure in e.g. Mouse, has Electrospun Fibrous Web Formed from Shape Memory Polymer and Near Infrared Dye with Excitation and Emission Wavelength. US2014303490-A1.

Matsumoto, H., Ishiguro, T., Konosu, Y., Minagawa, M., Tanioka, A., and Richau, K. (2012). Shape-memory properties of electrospun non-woven fabrics prepared from degradable polyesterurethanes containing poly(x-pentadecalactone) hard segments. Eur. Polym. J. 48, 1866-1874. doi:10.1016/j.eurpolymj.2012.07.008

Meng, Q. H., Hu, J. L., Zhu, Y., Lu, J., and Liu, Y. (2007). Morphology, phase separation, thermal and mechanical property differences of shape memory fibres prepared by different spinning methods. Smart Mater. Struct. 16, 1192-1197. doi:10.1088/0964-1726/16/4/030

Nagahama, K., Ueda, Y., Ouchi, T., and Ohya, Y. (2009). Biodegradable shape-memory polymers exhibiting sharp thermal transitions and controlled drug release. Biomacromolecules 10, 1789-1794. doi:10.1021/bm9002078

Peng, H., Zhou, S., Jiang, J., Guo, T., Zheng, X., and Yu, X. (2009). Pressure-induced crystal memory effect of spider silk proteins. J. Phys. Chem. B 113, 4636-4641. doi: $10.1021 /$ jp811461b

Rana, S., Kim, S. D., and Cho, J. W. (2013). Conducting core-sheath nanofibers for electroactive shape-memory applications. Polym. Adv. Technol. 24, 609-614. doi:10.1002/pat.3118

Reneker, D. H., and Chun, I. (1996). Nanometre diameter fibres of polymer, produced by electrospinning. Nanotechnology 7, 216-223. doi:10.1088/0957-4484/7/3/009 
Sahoo, N. G., Rana, S., Cho, J. W., Li, L., and Chan, S. H. (2010). Polymer nanocomposites based on functionalized carbon nanotubes. Prog. Polym. Sci. 35, 837-867. doi:10.1016/j.progpolymsci.2010.03.002

Sauter, T., Kratz, K., and Lendlein, A. (2012). Shape-memory properties of electrospun non-wovens prepared from amorphous polyetherurethanes under stressfree and constant strain conditions. MRS Proc., 1403(1), 105-112. doi:10.1557/ opl.2012.251

Schneider, T., Kohl, B., Sauter, T., Kratz, K., Lendlein, A., Ertel, W., et al. (2012). Influence of fiber orientation in electrospun polymer scaffolds on viability, adhesion and differentiation of articular chondrocytes. Clin. Hemorheol. Microcirc. 52, 325-336. doi:10.3233/CH-2012-1608

Shin, Y. M., Brenner, M. P., and Rutledge, G. C. (2001). Electrospinning: a whipping fluid jet generates submicron polymer fibers. Appl. Phys. Lett. 78, 1149-1151. doi:10.1063/1.1345798

Sun, Z. C., Zussman, E., Yarin, A. L., Wendorff, J. H., and Greiner, A. (2003). Compound core-shall polymer nanofibers by co-electrospinning. Adv. Mater. 15, 1929-1932. doi:10.1002/adma.200305136

Torbati, A. H., Mather, R. T., Reeder, J. E., and Mather, P. T. (2014). Fabrication of a light-emitting shape memory polymeric web containing indocyanine green. J. Biomed. Mater. Res. B Appl. Biomater. 102, 1236-1243. doi:10.1002/ jbm.b.33107

Tseng, L. F., Mathe, P. T., and Henderson, J. H. (2012). "A programmable shape-changing scaffold for regenerative medicine," in Bioengineering Conference (NEBEC), 2012 38th Annual Northeast (Philadelphia, PA: IEEE), 227-228. doi:10.1109/NEBC.2012.6207046

Tseng, L. F., Mather, P. T., and Henderson, J. H. (2013). Shape-memory-actuated change in scaffold fiber alignment directs stem cell morphology. Acta Biomater. 9, 8790-8801. doi:10.1016/j.actbio.2013.06.043

Xie, T. (2010). Tunable polymer multi-shape memory effect. Nature 464, 267-270. doi: $10.1038 /$ nature 08863

Zhang, F. H., Zhang, Z. C., Liu, Y. J., Cheng, W. L., Huang, Y. D., and Lenga, J. (2015). Thermosetting epoxy reinforced shape memory composite microfiber membranes: fabrication, structure and properties. Compos. Part A Appl. Sci. Manuf. 76, 54-61. doi:10.1016/j.compositesa.2015.05.004

Zhang, F. H., Zhang, Z. C., Liu, Y. J., and Leng, J. S. (2013a). "Fabrication of shape memory nanofibers by electrospinning method," in SPIE Proc (San Diego), 8687.

Zhang, F. H., Zhang, Z. C., Liu, Y. J., Lu, H. B., and Leng, J. S. (2013b). The quintuple-shape memory effect in electrospun nanofiber membranes. Smart Mater. Struct. 22, 085020. doi:10.1088/0964-1726/22/8/085020
Zhang, F. H., Zhang, Z. C., Liu, Y. J., and Leng, J. S. (2014a). Shape memory properties of electrospun Nafion nanofibers. Fibers Polym. 15, 534-539. doi:10.1007/ s12221-014-0534-Z

Zhang,F.H.,Leng,J.S.,Liu,Y.J., andZhang,Z.C.(2014b). Thermosetting-Thermoplastic Core-Shell Structured-Shape Memory Composite Fiber is Obtained by Providing Thermosetting Epoxy Resin as Core Layer and Thermoplastic Polycaprolactone as Shell Layer, and Recovering to Original Shape. CN104032409-A.

Zhang, F. H., Zhang, Z. C., Liu, Y. J., and Leng, J. S. (2014c). Electrospun nanofiber membranes for electrically activated shape memory nanocomposites. Smart Mater. Struct. 23, 065020. doi:10.1088/0964-1726/23/6/065020

Zhang, J. N., Ma, Y. M., Zhang, J. J., Dan, X., Yang, Q. L., Guan, J.-G., et al. (2011) Microfiber SMPU film affords quicker shape recovery than the bulk one. Mater. Lett. 65, 3639-3642. doi:10.1016/j.matlet.2011.06.083

Zhang, P. F., and Li, G. Q. (2013). Structural relaxation behavior of strain hardened shape memory polymer fibers for self-healing applications. J. Polym. Sci. Pol. Phys. 51, 966-977. doi:10.1002/polb.23295

Zhuo, H. T., Hu, J. L., and Chen, S. J. (2008a). Electrospun polyurethane nanofibres having shape memory effect. Mater. Lett. 62, 2074-2076. doi:10.1016/j. matlet.2007.11.018

Zhuo, H. T., Hu, J. L., Chen, S. J., and Yeung, L. Y. (2008b). Preparation of polyurethane nanofibers by electrospinning. J. Appl. Polym. Sci. 109, 406-411. doi:10.1002/app.28067

Zhuo, H. T., Hu, J. L., and Chen, S. J. (2011a). Study of the thermal properties of shape memory polyurethane nanofibrous nonwoven. J. Mater. Sci. 46, 3464-3469. doi:10.1007/s10853-011-5251-Z

Zhuo, H. T., Hu, J. L., and Chen, S. J. (2011b). Coaxial electrospun polyurethane core-shell nanofibers for shape memory and antibacterial nanomaterials. Express Polym. Lett. 5, 182-187. doi:10.3144/expresspolymlett.2011.16

Conflict of Interest Statement: The authors declare that the research was conducted in the absence of any commercial or financial relationships that could be construed as a potential conflict of interest.

Copyright (c) 2015 Zhang, Zhang, Zhou, Liu and Leng. This is an open-access article distributed under the terms of the Creative Commons Attribution License (CC BY). The use, distribution or reproduction in other forums is permitted, provided the original author(s) or licensor are credited and that the original publication in this journal is cited, in accordance with accepted academic practice. No use, distribution or reproduction is permitted which does not comply with these terms. 